\title{
Hydrodynamics in Two Dimensions and Vortex Theory *
}

\author{
C. Marchioro ${ }^{1}$ and M. Pulvirenti ${ }^{2}$ \\ 1 Dipartimento di Matematica, Libera Università di Trento, I-38050 Povo (Trento), Italy \\ 2 Istituto Matematico dell'Università di Roma, I-00100 Roma, Italy
}

\begin{abstract}
We consider the Navier-Stokes equation for a viscous and incompressible fluid in $\mathbb{R}^{2}$. We show that such an equation may be interpreted as a mean field equation (Vlasov-like limit) for a system of particles, called vortices, interacting via a logarithmic potential, on which, in addition, a stochastic perturbation is acting. More precisely we prove that the solutions of the Navier-Stokes equation may be approximated, in a suitable way, by finite dimensional diffusion processes with the diffusion constant related to the viscosity. As a particular case, when the diffusion constant is zero, the finite dimensional theory reduces to the usual deterministic vortex theory, and the limiting equation reduces to the Euler equation.
\end{abstract}

\section{Introduction}

In this paper we deal with an incompressible, viscid or inviscid fluid in two dimensions and study the connection between the equations governing the motion of such a fluid and the vortex theory. Furthermore we investigate some aspects of the hydrodynamical equations that, in particular, will suggest a quite natural proof of the existence and uniqueness of the solutions for a wide class of initial conditions.

It is well known that an incompressible and viscous two dimensional fluid, under the action of an external conservative field, is described by the following evolution equations

$$
\left.\begin{array}{c}
\frac{\partial \omega}{\partial t}(x, t)+(u \cdot \nabla) \omega(x, t)-v \Delta \omega(x, t)=0, \\
\omega(x, t)=\operatorname{curl} u(x, t)=\frac{\partial u_{2}}{\partial x_{1}}-\frac{\partial u_{1}}{\partial x_{2}}, \\
\nabla \cdot u=0
\end{array}\right\}
$$

* Partially supported by Italian CNR 
where $x=\left(x_{1}, x_{2}\right) \in \mathbb{R}^{2}, u=\left(u_{1}, u_{2}\right) \in \mathbb{R}^{2}$ is the velocities field, $v \geqq 0$ is the viscosity coefficient, $\nabla=\left(\frac{\partial}{\partial x_{1}}, \frac{\partial}{\partial x_{2}}\right), \Delta=\nabla \cdot \nabla$ is the Laplace operator.

In the following we shall denote Eq. (1.1) as the NS equation (Navier-Stokes) when $v>0$ and as the E equation (Euler) when $v=0$. Introducing the operator $\nabla^{\perp}$ $=\left(\frac{\partial}{\partial x_{2}}, \frac{\partial}{\partial x_{1}}\right)$, by virtue of $\nabla \cdot u=0$, one obtains

$$
u(x, t)=\int\left(\nabla^{\perp} g\right)(x-y) \omega(y, t) d y,
$$

where $g(r)=-\frac{1}{2 \pi} \ln r$ is the fundamental solution of the Poisson equation.

The E equation is related to the so-called vortex theory [1]. Let us consider a system of $N$ particles (vortices). To each particle is associated a vorticity intensity $a_{i} \in \mathbb{R}, i=1 \ldots N$. Denoting by $x_{i}$ the position of the $i^{\text {th }}$ vortex, we consider the following initial value problem:

$$
\left.\begin{array}{l}
\dot{x}_{i}(t)=\sum_{\substack{j \neq i \\
j=1}}^{N} a_{j}\left(\nabla^{\perp} g\right)\left(x_{i}(t)-x_{j}(t)\right), \\
x_{i}(0)=x_{i}, \quad i=1 \ldots N .
\end{array}\right\}
$$

If $x_{1} \ldots x_{N} \rightarrow x_{1}(t) \ldots x_{N}(t)$ is the solution of the initial value problem (1.3), (assuming that such a solution exists and is unique) then the signed measure $\omega_{t}^{N}(d x)$ $=\sum_{i=1}^{N} a_{i} \delta_{x_{i}(t)}(d x)$ [here $\delta_{\bar{x}}(d x)$ denotes the Dirac measure based on $\bar{x}$ ] satisfies the following identity:

$$
\frac{d}{d t} \omega_{t}^{N}(f)=\omega_{t}^{N}\left[\left(u_{0} \cdot \nabla\right) f\right]
$$

for all sufficiently regular $f$, where

$$
\begin{gathered}
u_{0}(x, t)=\int\left(\nabla^{\perp} g\right)(x-y) X(\{x \neq y\}) \omega_{t}^{N}(d y), \\
X(\{x \neq y\})= \begin{cases}1 & \text { if } x \neq y \\
0 & \text { otherwise }\end{cases}
\end{gathered}
$$

In fact, by a direct computation,

$$
\begin{aligned}
\frac{d}{d t} \omega_{t}^{N}(f) & =\frac{d}{d t} \sum_{i=1}^{N} a_{i} f\left(x_{i}(t)\right) \\
& =\sum_{i=1}^{N} a_{i}(\nabla f)\left(x_{i}(t)\right) \cdot \dot{x}_{i}(t) \\
& =\sum_{i=1}^{N} a_{i} \sum_{\substack{j \neq i \\
j=1}}^{N} a_{j}(\nabla f)\left(x_{i}(t)\right) \cdot\left(\nabla^{\perp} g\right)\left(x_{i}(t)-x_{j}(t)\right) \\
& =\omega_{t}^{N}\left[\left(u_{0} \cdot \nabla\right) f\right] .
\end{aligned}
$$


Thus Eq. (1.4) is a weak version of the E equation, except that the self-energy factor (that would give an infinite contribution) is neglected in the definition of the velocity field.

The problem of finding a rigorous connection between the evolution problems (1.3) and (1.4) in case $v=0$ arises quite naturally. What is expected to be true is the following. Let $\omega$ be a sufficiently smooth initial condition for the Equation and suppose that $\omega$ is approximated, in some sense, by $\omega^{N}=\sum_{i=1}^{N} a_{i} \delta_{x_{2}}\left[x_{i}\right.$ initial conditions for the initial value problem (1.3)]. Then $\omega_{t}^{N}$ would approximate $\omega_{t}$, the solution of the Equation with initial datum $\omega$.

Although such an approximation problem has been widely investigated for numerical purposes (see [2] for example), as far as we know it has not been solved (see [3, p. 110]). In this paper we treat this problem, also in the presence of viscosity, giving a precise connection between the NS equation and a generalization of the vortex theory.

We describe the basic idea. The E equation in two dimensions is described by characteristics. In fact, by a direct inspection of the equation, we realize that each molecule of vorticity evolves under the force field generated by all other molecules. Such an idea has been used by Kato (and previously by other authors) to obtain an existence and uniqueness theorem for classical solutions of such an equation [4]. The presence of the Laplace operator in the NS equation suggests the use of stochastic characteristics in place of deterministic ones. Such a natural idea has already been used in [5] to give a derivation of the NS equation ${ }^{1}$. Other attempts in this direction, different and independent from ours, are going to be developed [6].

Our approach is the following. We first deal with a regularized version of the initial value problem associated to (1.1), i.e. we replace $g$ by $g_{\varepsilon}$, a smooth function different from $g$ in an $\varepsilon$-sphere around the origin. For such a new initial value problem we prove existence and uniqueness of the solutions, by the use of the contraction principle (Sect. 2).

The techniques used in Sect. 2 are inspired by the approach proposed by Dobrushin in [7] for the Vlasov equation that is very similar to the E equation. The only difference with our case is that we deal with stochastic differential equations in place of ordinary differential problems, in order to take into account the viscosity term. The main part of the results of this section have also been obtained by McKean [8] in a different physical context.

In Sect. 3 we remove the cutoff, proving that the above solutions are a convergent sequence when $\varepsilon \rightarrow 0$. In particular we obtain, for a large class of initial conditions, an existence and uniqueness theorem by means of a constructive procedure.

In Sect. 4 we show that a system of $N$ vortices, interacting via a potential $g_{\varepsilon}$, under a stochastic perturbation, has a solution which converges to the solution of the NS equation in the limit $N \rightarrow \infty$ and $\varepsilon=\varepsilon(N) \rightarrow 0$, once the initial datum of the vortex systems approach the initial datum of the NS equation, at time zero.

1 It is also the basis of a numerical method due to Chorin for the study of slightly viscous flow: J. Fluid Mech. 57, 785 (1973) 
All the results we obtain hold for the NS equation as well as for the E equation, all our estimates being independent of $v$, with the exception of Sect. 4 in which a stronger result is obtained for the deterministic case.

We finally remark that from the point of view of our approach, we are interested in weak solutions of our initial value problems. In the case of sufficiently smooth initial conditions, the uniqueness guarantees that we actually obtain classical solutions, which, as it is known, do exist.

\section{Regularized Problem}

Let $M$ be a separable and complete metric space, with a uniformly bounded metric $d: M \times M \rightarrow \mathbb{R}^{+}$. Let $\mathscr{M}(M)$ be the space of the Borel, signed measures on $M$ and $\mathscr{M}^{+}(M)$ its positive part. For all $\mu \in \mathscr{M}(M)$ let us denote $\mu^{+}$and $\mu^{-}$the positive and negative part of $\mu$ according to the Jordan decomposition of $\mu$ and $\|\mu\|=\int d \mu^{+}$ $+\int d \mu^{-}$its total variation. We introduce the following spaces

$$
\begin{aligned}
\mathscr{M}^{+}(M ; a) & =\left\{\mu \in \mathscr{M}^{+}(M) \mid\|\mu\|=a\right\}, \\
\mathscr{M}^{+}(M ; a, b) & =\left\{\mu \in \mathscr{M}(M) \mid\left\|\mu^{+}\right\|=a,\left\|\mu^{-}\right\|=b\right\} .
\end{aligned}
$$

A metric topology can be given on $\mathscr{M}(M, a, b)$ in the following way. Let $\mu, v \in \mathscr{M}^{+}(M, 1)$ and $\mathscr{C}(\mu, v)$ be the set of all joint representations of $\mu$ and $v$. We recall that a joint representation $P$ is a probability measure on $M \times M$ such that

$$
\begin{aligned}
& \int P(d x, d y) f(x)=\int \mu(d x) f(x), \\
& \int P(d x, d y) f(y)=\int \nu(d y) f(y),
\end{aligned}
$$

for all Borel functions $f$.

We define:

$$
R_{1}(\mu, v)=\inf _{P \in \mathscr{C}(\mu, v)} \int P(d x, d y) d(x, y)
$$

$R_{1}$ is a metric on $\mathscr{M}^{+}(1)$ and induces a topology that is equivalent to the weak convergence topology [9]. The metric $R_{1}$ is called the Kantorovich-Rubinstein $(\mathrm{KR})$ distance and will play a basic role in our analysis.

For all $\mu, v \in \mathscr{M}^{ \pm}(M, a)$ we define

$$
\hat{R}(\mu, v)=R_{1}\left(\frac{\mu}{a}, \frac{v}{a}\right)
$$

and if $\mu, v \in \mathscr{M}(M, a, b)$ we put

$$
\hat{R}(\mu, v)=\hat{R}\left(\mu^{+}, v^{+}\right)+\hat{R}\left(\mu^{-}, v^{-}\right) .
$$

The topology induced by the metric (2.5) is equivalent to the weak convergence topology on $\mathscr{M}(M, a, b)$ and makes it separable and complete. We specialize the above considerations by putting $M=\mathbb{R}^{2}, d(x, y)=\min (|x-y|, 1),|x|=\sqrt{\sum_{\alpha=1}^{2}\left|x_{\alpha}\right|^{2}}$. Since the metric $d$ is topologically equivalent to the usual metric, the topology induced by $\hat{R}$ in $\mathscr{M}\left(\mathbb{R}^{2} ; a, b\right)$ is equivalent to the usual weak convergence topology. 
For notational simplicity we put $\mathscr{M}^{+}=\mathscr{M}^{+}\left(\mathbb{R}^{2}\right), \mathscr{M}^{+}(a)=\mathscr{M}^{+}\left(\mathbb{R}^{2}, a\right), \mathscr{M}(a, b)$ $=\mathscr{M}\left(\mathbb{R}^{2} ; a, b\right)$ etc.

With the above tools we shall treat a regularized version of the NS equation. Let $g$ be the fundamental solution of the Poisson equation in $\mathbb{R}^{2}$ and $g_{\varepsilon}$ defined as $g_{\varepsilon}(r)=g(r)$ if $|r| \geqq \varepsilon$ and arbitrarily extended to an even $C^{2}\left(\mathbb{R}^{1}\right)$ function such that $\left|g_{\varepsilon}^{\prime}(r)\right| \leqq\left|g^{\prime}(r)\right|,\left|g_{\varepsilon}^{\prime \prime}(r)\right| \leqq\left|g^{\prime \prime}(r)\right|$. Defining $K_{\varepsilon}(x)=\left(\nabla^{\perp} g_{\varepsilon}\right)(|x|), x \in \mathbb{R}^{2}$ we have:

Theorem 2.1. Let $\omega \in \mathscr{M}\left(a^{+}, a^{-}\right)$and $v \geqq 0$. There exists a unique function $\mathbb{R}^{+} \ni t \rightarrow \omega_{t}^{\varepsilon} \in \mathscr{M}\left(a^{+}, a^{-}\right)$with the following properties: for all $f \in \mathscr{C}^{2}\left(\mathbb{R}^{2}\right)$, such that $|\nabla f|$ and $\Delta f \in L_{\infty}\left(\mathbb{R}^{2}\right)$

$$
\left.\begin{array}{rl}
\frac{d}{d t} \omega_{t}^{\varepsilon}(f) & =\omega_{t}^{\varepsilon}\left[\left(u_{\varepsilon} \cdot \nabla\right) f\right]+v \omega_{t}^{\varepsilon}(\Delta f) \\
\omega_{0}^{\varepsilon} & =\omega,
\end{array}\right\}
$$

where

$$
u_{\varepsilon}(x, t)=\int K_{\varepsilon}(x-y) \omega_{t}^{\varepsilon}(d y) .
$$

Moreover, for all Borel sets $A \subset \mathbb{R}^{2}$,

$$
\omega_{t}^{\varepsilon}(A)=\int P_{t}^{\varepsilon}(A \mid y) \omega(d y),
$$

where $P_{t}^{\varepsilon}(\cdot \mid y)$ are the transition probabilities of the diffusion process, solution of the stochastic differential equation

$$
d x(t)=u_{\varepsilon}(x(t), t) d t+\sigma d w(t)
$$

with $\frac{\sigma^{2}}{2}=v$

Proof. Let $T>0$ and $\mathscr{M}\left(a^{+}, a^{-} ; T\right)$ the space of the continuous functions

$$
[0, T] \ni t \rightarrow \mu_{t} \in \mathscr{M}\left(a^{+}, a^{-}\right) .
$$

$\mathscr{M}\left(a^{+}, a^{-} ; T\right)$ is a complete metric space with metric function given by

$$
R_{T}\left(\tilde{\mu}_{1}, \tilde{\mu}_{2}\right)=\sup _{t \in[0, T]} \hat{R}\left(\mu_{t}^{1}, \mu_{t}^{2}\right)
$$

where $\tilde{\mu}_{i}=\left\{\mu_{t}^{i}\right\}_{t \in[0, T]}$.

Let $(\Omega, \Sigma, P)$ be a probability space (such space will not play any significant role in the rest of the section) and $w$ a standard Wiener process on it.

We consider the processes solutions of the following stochastic differential equation

$$
d x_{i}(t)=u_{\varepsilon}^{i}(x ;(t), t) d t+\sigma d w(t),
$$

where

$$
u_{\varepsilon}^{i}(x, t)=\int K_{\varepsilon}(x-y) \mu_{t}^{i}(d y)
$$

and $\tilde{\mu}_{i} \equiv\left\{\mu_{t}^{i}\right\}_{t \in[0, T]} \in \mathscr{M}\left(a^{+}, a^{-} ; T\right), i=1,2$. 
Denoting by $x_{i}(t, x)$ the unique process solution of (2.12) starting almost surely from $x$ at time zero, and by $E$ the expectation with respect to $P$, we have the following estimate

$$
E\left[d\left(x_{1}(t, x), x_{2}(t, x)\right)\right] \leqq\left(a L_{\varepsilon} \exp 2 a L_{\varepsilon} T\right) \int_{0}^{t} d s \hat{R}\left(\mu_{s}^{1}, \mu_{s}^{2}\right),
$$

where $L_{\varepsilon}=\max \left(\tilde{L}_{\varepsilon}, 2 \max K_{\varepsilon}\right), \quad \tilde{L}_{\varepsilon}$ is the Lipschitz constant of $K_{\varepsilon}$ and $a=\max \left(a^{+}, a^{-}\right)$.

Proof of (2.14). With the above notation we have $\left|K_{\varepsilon}\left(x_{1}-y\right)-K_{\varepsilon}\left(x_{2}-y\right)\right|$ $\leqq L_{\varepsilon} d\left(x_{1}, x_{2}\right)$. Hence, $\langle P=1\rangle$,

$$
\begin{aligned}
& d\left(x_{1}(t, x), x_{2}(t, x)\right) \leqq\left|x_{1}(t, x)-x_{2}(t, x)\right| \\
& \leqq \int_{0}^{t} d s\left|u_{\varepsilon}^{1}\left(x_{1}(s, x), s\right)-u_{\varepsilon}^{2}\left(x_{2}(s, x), s\right)\right| \\
& \leqq \int_{0}^{t} d s\left\{\left|u_{\varepsilon}^{1}\left(x_{1}(s, x), s\right)-u_{\varepsilon}^{1}\left(x_{2}(s, x), s\right)\right|+\left|u_{\varepsilon}^{1}\left(x_{2}(s, x), s\right)-u_{\varepsilon}^{2}\left(x_{2}(s, x), s\right)\right|\right\} \\
& \leqq \int_{0}^{t} d s\left\{\left|\int \mu_{s}^{1}(d y)\left[K_{\varepsilon}\left(x_{1}(s, x)-y\right)-K_{\varepsilon}\left(x_{2}(s, x)-y\right)\right]\right|\right. \\
& \left.+\left|\int K_{\varepsilon}\left(x_{2}(s, x)-y\right)\left[\mu_{s}^{1}(d y)-\mu_{s}^{2}(d y)\right]\right|\right\} \\
& \leqq 2 a L_{\varepsilon} \int_{0}^{t} d s d\left(x_{1}(s, x), x_{2}(s, x)\right) \\
& \int_{0}^{t} d s\left|\int K_{\varepsilon}\left(x_{2}(s)-y\right)\left(\left\{\mu_{s}^{1+}(d y)-\mu_{s}^{2+}(d y)\right\}+\left\{\mu_{s}^{2-}(d y)-\mu_{s}^{2-}(d y)\right\}\right)\right| \\
& \leqq 2 a L_{\varepsilon} \int_{0}^{t} d s d\left(x_{1}(s, x), x_{2}(s, x)\right) \\
& +\int_{0}^{t} d s\left\{a^{+} \int P_{+}^{s}\left(d y_{1}, d y_{2}\right)\left|K_{\varepsilon}\left(x_{2}(s, x)-y_{1}\right)-K_{\varepsilon}\left(x_{2}(s, x)-y_{2}\right)\right|\right. \\
& \left.+a^{-} \int P_{-}^{s}\left(d y_{1}, d y_{2}\right)\left|K_{\varepsilon}\left(x_{2}(s, x)-y_{1}\right)-K_{\varepsilon}\left(x_{2}(s, x)-y_{2}\right)\right|\right\},
\end{aligned}
$$

for all $P_{ \pm}^{s} \in \mathscr{C}\left(\frac{\mu_{s}^{2 \pm}}{a^{ \pm}}, \frac{\mu_{s}^{2 \pm}}{a^{ \pm}}\right)$. Thus

$$
\begin{aligned}
E\left[d\left(x_{1}(t, x), x_{2}(t, x)\right)\right] \leqq & 2 a L_{\varepsilon}\left\{\int_{0}^{t} d s E\left[d\left(x_{1}(t, x), x_{2}(t, x)\right)\right]\right. \\
& \left.+\frac{1}{2} \int_{0}^{t} d s \hat{R}\left(\mu_{s}^{1}, \mu_{s}^{2}\right)\right\}
\end{aligned}
$$

that implies (2.14).

We define the following map $\mathscr{M}\left(a^{+}, a^{-} ; T\right) \rightarrow \mathscr{M}\left(a^{+}, a^{-} ; T\right)$,

$$
\tilde{\mu}=\left\{\mu_{t}\right\}_{t \in[0, T]} \rightarrow S^{\tilde{\mu}} \omega=\left\{S_{t}^{\tilde{\mu}} \omega\right\}_{t \in[0, T]}:\left(S_{t}^{\tilde{\mu}} \omega\right)(A)=\int P_{t}^{\tilde{\mu}}(A \mid y) \omega(d y),
$$


where $P_{t}^{\mu}(\cdot \mid y)$ are the transition probabilities of the process solution of $(2.12)$ with $\tilde{\mu}_{i}$ replaced by $\tilde{\mu}$. Obviously $S_{t}^{\tilde{\mu}} \omega \in \mathscr{M}\left(a^{+}, a^{-}\right)$. Moreover, for all $f \in C_{2}\left(\mathbb{R}^{2}\right)$ such that $|\nabla f|$ and $\Delta f$ are bounded, the following holds [10]:

$$
\left.\begin{array}{c}
\frac{d}{d t}\left(S_{t}^{\tilde{\mu}} \omega\right)(f)=\left(S_{t}^{\tilde{\mu}} \omega\right)\left[\left(u_{\varepsilon}^{\tilde{\mu}} \cdot \nabla\right) f\right]+\frac{\sigma^{2}}{2}\left(S_{t}^{\tilde{\mu}} \omega\right)(\Delta f), \\
S_{0}^{\tilde{\mu}} \omega=\omega, \\
u_{\varepsilon}^{\tilde{\mu}}(x, t)=\int K_{\varepsilon}(x-y) \mu_{t}(d y) .
\end{array}\right\}
$$

We prove that the map $\tilde{\mu} \rightarrow S^{\tilde{\mu}} \omega$ is a contraction in $\mathscr{M}\left(a^{+}, a^{-} ; T\right)$ for small $T$ as consequence of (2.14). Putting $\omega_{t}^{i}=S_{t}^{\tilde{\mu}_{i}} \omega$, we define $Q_{t}^{ \pm}\left(d x_{1}, d x_{2}\right)$ via the relations:

$$
\int Q_{t}^{ \pm}\left(d x_{1}, d x_{2}\right) f\left(x_{1}, x_{2}\right)=\frac{1}{a^{ \pm}} \int \omega^{ \pm}(d x) E\left[f\left(x_{1}(t, x), x_{2}(t, x)\right]\right.
$$

Then $Q_{t}^{ \pm} \in \mathscr{C}\left(\frac{\omega^{1 \pm}}{a^{ \pm}}, \frac{\omega^{2 \pm}}{a^{ \pm}}\right)$. Thus, integrating both members of (2.14) with $\frac{\omega^{ \pm}}{a^{ \pm}}(d x)$ and summing

$$
\hat{R}\left(\omega_{t}^{1}, \omega_{t}^{2}\right) \leqq\left(2 a L_{\varepsilon} \exp 2 a L_{\varepsilon} T\right) \int_{0}^{t} d s \hat{R}\left(\mu_{s}^{1}, \mu_{s}^{2}\right) .
$$

From (2.21) we obtain the existence of a unique fixed point of the map $\tilde{\mu} \rightarrow S^{\tilde{\mu}} \omega$ for small $T$ and hence a unique solution of the initial value problem (2.6). Such a solution may be extended to arbitrary times so the proof is complete.

Remark. Theorem 2.1 deals in particular with the regularized Euler problem. In this case

$$
P_{t}^{\varepsilon}(d x \mid y)=\delta\left(T_{t}^{\varepsilon} y-x\right) d x
$$

where $y \rightarrow T_{t}^{\varepsilon} y$ is the solution of the initial value problem

$$
\left.\begin{array}{rl}
\frac{d}{d t} T_{t}^{\varepsilon} y & =u_{\varepsilon}\left(T_{t}^{\varepsilon} y, t\right), \\
T_{0}^{\varepsilon} y & =y .
\end{array}\right\}
$$

Proposition 2.1. Let $\tilde{\mu} \in \mathscr{M}\left(a^{+}, a^{-} ; T\right), \omega_{i} \in \mathscr{M}\left(a^{+}, a^{-}\right), i=1,2$. Then

$$
\hat{R}\left(S_{t}^{\tilde{\mu}} \omega_{1}, S_{t}^{\tilde{\mu}} \omega_{2}\right) \leqq\left(\exp 2 a L_{\varepsilon} t\right) \hat{R}\left(\omega_{1}, \omega_{2}\right)
$$

Proof. Denoting by $x(t, x)$ the process solution of

$$
d x(t)=u_{\varepsilon}^{\tilde{\mu}}(x(t), t) d t+\sigma d w(t)
$$

starting almost surely from $x$ at time zero, we have, $\langle P=1\rangle$,

$$
\begin{aligned}
& d(x(t, x), x(t, y)) \leqq|x-y|+\int_{0}^{t} d s\left|u_{\varepsilon}^{\tilde{\mu}}(x(s, x), s)-u_{\varepsilon}^{\tilde{\mu}}(x(s, y), s)\right| \\
& \quad \leqq|x-y|+\int_{0}^{t} d s\left|\int \mu_{s}(d z)\left\{K_{\varepsilon}(x(s, x)-z)-K_{\varepsilon}(x(s, y)-z)\right\}\right| \\
& \quad \leqq|x-y|+2 L_{\varepsilon} a \int_{0}^{t} d s d(x(s, x), x(s, y))
\end{aligned}
$$


and hence

$$
\begin{aligned}
& E[d(x(t, x), x(t, y))] \leqq|x-y| \exp 2 a L_{\varepsilon} t, \quad \text { that implies } \\
& E[d(x(t, x), x(t, y))] \leqq d(x, y) \exp 2 a L_{\varepsilon} t
\end{aligned}
$$

because $E[d(x(t, x), x(t, y))] \leqq 1$.

We define $P_{t}^{ \pm}$by

$$
\int P_{t}^{ \pm}(d x, d y) f(x, y)=\int P^{ \pm}(d x, d y) E[f(x(t, x), x(t, y))]
$$

for $P^{ \pm} \in \mathscr{C}\left(\frac{\omega_{1}^{ \pm}}{a^{ \pm}}, \frac{\omega_{2}^{ \pm}}{a^{ \pm}}\right)$. By direct inspection

$$
P_{t}^{ \pm} \in \mathscr{C}\left(\frac{\left(S_{t}^{\tilde{\mu}} \omega_{1}\right)^{ \pm}}{a^{ \pm}}, \frac{\left(S_{t}^{\tilde{\mu}} \omega_{2}\right)^{ \pm}}{a^{ \pm}}\right)
$$

Thus the thesis follows by integrating (2.29) with $P^{ \pm}$.

The next theorem describes the continuity property of the regularized solutions with respect to the initial conditions.

Theorem 2.2. Let $\tilde{\omega}^{\varepsilon}$ and $\tilde{\xi}^{\varepsilon} \in \mathscr{M}\left(a^{+}, a^{-} ; T\right)$ be two solutions of the initial value problem (2.6) with initial conditions $\omega$ and $\xi$ respectively. Then

$$
R_{T}\left(\tilde{\omega}^{\varepsilon}, \tilde{\xi}^{\varepsilon}\right) \leqq \hat{R}(\omega, \xi) \exp \left[2 a L_{\varepsilon}\left(T+e^{2 a L_{\varepsilon} T}\right)\right] .
$$

Proof.

$$
\hat{R}\left(\omega_{t}^{\varepsilon}, \xi_{t}^{\varepsilon}\right) \leqq \hat{R}\left(\omega_{t}^{\varepsilon}, S_{t}^{\tilde{\xi}^{\varepsilon}} \omega\right)+\hat{R}\left(S_{t}^{\tilde{\xi}^{\varepsilon}} \omega, \xi_{t}^{\varepsilon}\right)
$$

By virtue of (2.21)

$$
\hat{R}\left(\omega_{t}^{\varepsilon}, \xi_{t}^{\varepsilon}\right) \leqq\left(2 a L_{\varepsilon} \exp 2 a L_{\varepsilon} t\right) \int_{0}^{t} d s \hat{R}\left(\omega_{s}^{\varepsilon}, \xi_{s}^{\varepsilon}\right),
$$

and by Proposition 2.1

$$
\hat{R}\left(S_{t}^{\tilde{\xi}^{\varepsilon}} \omega, \xi_{t}^{\varepsilon}\right) \leqq\left(\exp 2 a L_{\varepsilon} t\right) \hat{R}(\omega, \xi) .
$$

The thesis follows combining the above estimates.

\section{Construction of the Solutions}

In this section we shall obtain weak solutions of the NS and E equations as limit of the regularized solutions as $\varepsilon \rightarrow 0$.

Theorem 3.1. Let $\omega \in L_{1} \cap L_{\infty}\left(\mathbb{R}^{2}\right), \omega d x \in \mathscr{M}\left(a^{+}, a^{-}\right), v \geqq 0$. There exists a unique solution $\omega \rightarrow \omega_{t} \in L_{1} \cap L_{\infty}\left(\mathbb{R}^{2}\right), \omega_{t} d x \in \mathscr{M}\left(a^{+}, a^{-}\right)$of the initial value problem (weak form of the NS and E equations)

$$
\left.\begin{array}{rl}
\frac{d}{d t} \omega_{t}(f) & =\omega_{t}[(u \cdot \nabla) f]+v \omega_{t}(\Delta f) \\
\omega_{0} & =\omega
\end{array}\right\}
$$


for all $f \in C_{2}\left(\mathbb{R}^{2}\right)$, such that $|\nabla f|, \Delta f \in L_{\infty}\left(\mathbb{R}^{2}\right)$, where

$$
\begin{gathered}
u(x, t)=\int K(x-y) \omega_{t}(y) d y \\
K(x)=\left(\nabla^{\perp} g\right)(|x|), \quad x \in \mathbb{R}^{2} .
\end{gathered}
$$

Moreover

$$
\omega_{t}(f)=\lim _{\varepsilon \rightarrow 0} \omega_{t}^{\varepsilon}(f)
$$

for all $f \in C \cap L_{\infty}\left(\mathbb{R}^{2}\right)$ and the above limit holds uniformly in $v$ and $t$ on compact sets.

Finally, there exists a unique solution of the following stochastic differential equation

$$
d x(t)=u(x(t), t) d t+\sigma d w(t)
$$

once specified the distribution of the random variable $x(\cdot)$, and denoting by $P_{t}(d x \mid y)$ the transition probabilities associated to the solution of $(3.4)$, it results $\left(v=\frac{\sigma^{2}}{2}\right)$

$$
\int_{A} \omega_{t}(x) d x=\int P_{t}(A \mid y) \omega(y) d y .
$$

We need a preliminary lemma (essentially Lemma 1.4 in [4]), whose proof will be postponed to the end of the section.

Lemma 3.1. Let $t \rightarrow \omega_{t}(x)$ be a family of $L_{1} \cap L_{\infty}\left(\mathbb{R}^{2}\right)$ functions such that

$$
\sup _{t \in[0, T]}\left\|\omega_{t}\right\|_{\infty} \leqq A_{1}, \quad \sup _{t \in[0, T]}\left\|\omega_{t}\right\|_{1} \leqq A_{2}
$$

Then, for all $\varepsilon \geqq 0$ and $t \in[0, T]$,

$$
\begin{gathered}
\int\left|\omega_{t}(y)\right|\left|K_{\varepsilon}(x-y)\right| d y \leqq c_{1}\left(A_{1}+A_{2}\right), \\
\int\left|\omega_{t}(y)\right|\left|K_{\varepsilon}(x-y)-K_{\varepsilon}\left(x^{\prime}-y\right)\right| d y \leqq 2 c_{1}\left(A_{1}+A_{2}\right) \varphi\left(x, x^{\prime}\right),
\end{gathered}
$$

where

$$
\begin{gathered}
\varphi\left(x, x^{\prime}\right)=\tilde{\varphi}\left(\left|x-x^{\prime}\right|\right), \\
\tilde{\varphi}(r)=\left\{\begin{array}{lll}
r(1-\ln r) & \text { if } & 0<r<1 \\
1 & \text { if } & r \geqq 1 .
\end{array}\right.
\end{gathered}
$$

Proof of Theorem 3.1. We first observe, by general properties of the diffusion processes with smooth drift, (see e.g. [10]) that, if $\omega \in L_{1} \cap L_{\infty}\left(\mathbb{R}^{2}\right)$, then $\omega_{t}^{\varepsilon} \in L_{1} \cap L_{\infty}\left(\mathbb{R}^{2}\right)$ and $\left\|\omega_{t}^{\varepsilon}\right\|_{\infty} \leqq\|\omega\|_{\infty},\left\|\omega_{t}^{\varepsilon}\right\|_{1}=\|\omega\|_{1}$.

Let $x^{\varepsilon}(t), x^{\varepsilon^{\prime}}(t)$ be two processes constructed in Theorem 2.1, with $\varepsilon>\varepsilon^{\prime}>0$, and starting from $x \in \mathbb{R}^{2}$ at time zero almost surely, then $\langle P=1\rangle$,

$$
\begin{aligned}
d\left(x^{\varepsilon}(t), x^{\varepsilon^{\prime}}(t)\right) \leqq & \int_{0}^{t} d s\left|u_{\varepsilon}\left(x^{\varepsilon}(s), s\right)-u_{\varepsilon^{\prime}}\left(x^{\varepsilon^{\prime}}(s), s\right)\right| \\
\leqq & \int_{0}^{t} d s\left\{\left|u_{\varepsilon}\left(x^{\varepsilon}(s), s\right)-u_{\varepsilon}\left(x^{\varepsilon^{\prime}}(s), s\right)\right|\right. \\
& \left.+\left|u_{\varepsilon}\left(x^{\varepsilon^{\prime}}(s), s\right)-u_{\varepsilon^{\prime}}\left(x^{\varepsilon^{\prime}}(s), s\right)\right|\right\} .
\end{aligned}
$$


By virtue of Lemma 3.1

where

$$
\left|u_{\varepsilon}\left(x^{\varepsilon}(s), s\right)-u_{\varepsilon}\left(x^{\varepsilon^{\prime}}(s), s\right)\right| \leqq c(\omega) \varphi\left(x^{\varepsilon}(s), x^{\varepsilon^{\prime}}(s)\right),
$$

$$
c(\omega)=2 c_{1}\left(\|\omega\|_{1}+\|\omega\|_{\infty}\right) .
$$

Furthermore

$$
\begin{aligned}
& \left|u_{\varepsilon}\left(x^{\varepsilon^{\prime}}(s), s\right)-u_{\varepsilon^{\prime}}\left(x^{\varepsilon^{\prime}}(s), s\right)\right| \\
& =\left|\int d y \omega_{s}^{\varepsilon}(y) K_{\varepsilon}\left(x^{\varepsilon^{\prime}}(s)-y\right)-\int d y \omega_{s}^{\varepsilon^{\prime}}(y) K_{\varepsilon^{\prime}}\left(x^{\varepsilon^{\prime}}(s)-y\right)\right| \\
& \leqq\left|\int d y\left(\omega_{s}^{\varepsilon}(y)-\omega_{s}^{\varepsilon^{\prime}}(y)\right) K_{\varepsilon}\left(x^{\varepsilon^{\prime}}(s)-y\right)\right| \\
& +1 \int d y \omega_{s}^{\varepsilon^{\prime}}(y)\left\{K_{\varepsilon}\left(x^{\varepsilon^{\prime}}(s)-y\right)-K_{\varepsilon^{\prime}}\left(x^{\varepsilon^{\prime}}(s)-y\right)\right\} \\
& \leqq\left|\int d y\left(\omega_{s}^{\varepsilon}(y)-\omega_{s}^{\varepsilon^{\prime}}(y)\right) K_{\varepsilon}\left(x^{\varepsilon^{\prime}}(s)-y\right)\right| \\
& +c_{2}\|\omega\|_{\infty} \int_{|y| \leqq \varepsilon} d y|y|^{-1} \text {, }
\end{aligned}
$$

for some $c_{2}>0$. Hence, using the notation $E_{x}\left[f\left(x^{\varepsilon}(t)\right)\right]=E\left[f\left(x^{\varepsilon}(t, x)\right)\right]$, for some $c_{3}>0$, we have

$$
\begin{aligned}
& \int \frac{\omega^{+}(x)}{a^{+}} E_{x}\left[d\left(x^{\varepsilon}(t), x^{\varepsilon^{\prime}}(t)\right)\right] d x \\
& \leqq \int_{0}^{t} d s\left\{c_{3}\|\omega\|_{\infty} \varepsilon+c(\omega) \int \frac{\omega^{+}(x)}{a^{+}} d x E_{x}\left[\varphi\left(x^{\varepsilon}(s), x^{\varepsilon^{\prime}}(s)\right)\right]\right\} \\
& \quad+\int d x \frac{\omega^{+}(x)}{a^{+}} E_{x}\left[\left|\int d y\left(\omega_{s}^{\varepsilon}(y)-\omega_{s}^{\varepsilon^{\prime}}(y)\right) K_{\varepsilon}\left(x^{\varepsilon^{\prime}}(s)-y\right)\right|\right] .
\end{aligned}
$$

Since

$$
\int d y \omega_{s}^{\varepsilon}(y) K_{\varepsilon}\left(x^{\varepsilon^{\prime}}(s)-y\right)=\int d y \omega(y) E_{y}\left[K_{\varepsilon}\left(x^{\varepsilon^{\prime}}(s)-y^{\varepsilon}(s)\right)\right],
$$

we obtain

$$
\begin{aligned}
& E_{x}\left[\left|\int d y\left(\omega_{s}^{\varepsilon}(y)-\omega_{s}^{\varepsilon^{\prime}}(y)\right) K_{\varepsilon}\left(x^{\varepsilon^{\prime}}(s)-y\right)\right|\right] \\
& \quad=E_{x}\left[\left|\int d y \omega(y) E_{y}\left[K_{\varepsilon}\left(x^{\varepsilon^{\prime}}(s)-y^{\varepsilon}(s)\right)-K_{\varepsilon}\left(x^{\varepsilon^{\prime}}(s)-y^{\varepsilon^{\prime}}(s)\right)\right]\right|\right] \\
& \quad \leqq a \int d y\left\{\frac{\omega^{+}(y)}{a^{+}}+\frac{\omega^{-}(y)}{a^{-}}\right\} E_{x} E_{y}\left[\left|K_{\varepsilon}\left(x^{\varepsilon^{\prime}}(s)-y^{\varepsilon}(s)\right)-K_{\varepsilon}\left(x^{\varepsilon^{\prime}}(s)-y^{\varepsilon^{\prime}}(s)\right)\right|\right] .
\end{aligned}
$$

Thus

$$
\begin{aligned}
\int d x & \frac{\omega^{+}(x)}{a^{+}}(3.16) \leqq a \int d y\left\{\frac{\omega^{+}(y)}{a^{+}}+\frac{\omega^{-}(y)}{a^{-}}\right\} \\
\cdot & E_{y}\left[\int d x \frac{\omega^{+}(x)}{a^{+}} E_{x}\left(\left|K_{\varepsilon}\left(x^{\varepsilon^{\prime}}(s)-y^{\varepsilon}(s)\right)-K_{\varepsilon}\left(x^{\varepsilon^{\prime}}(s)-y^{\varepsilon^{\prime}}(s)\right)\right|\right)\right] \\
= & a \int d y\left\{\frac{\omega^{+}(y)}{a^{+}}+\frac{\omega^{-}(y)}{a^{-}}\right\} \cdot E_{y} \\
& \cdot\left[\int d x \frac{\omega_{s}^{\varepsilon^{\prime}}(x)}{a^{+}}\left|K_{\varepsilon}\left(x-y^{\varepsilon}(s)\right)-K_{\varepsilon}\left(x-y^{\varepsilon^{\prime}}(s)\right)\right|\right] \\
\leqq & a c\left(\frac{\omega^{+}}{a^{+}}\right) \int d y\left\{\frac{\omega^{+}(y)}{a^{+}}+\frac{\omega^{-}(y)}{a^{-}}\right\} \cdot E_{y}\left[\varphi\left(y^{\varepsilon}(s), y^{\varepsilon^{\prime}}(s)\right)\right] .
\end{aligned}
$$


Defining, finally:

$$
\begin{aligned}
& Y_{1}\left(\varepsilon, \varepsilon^{\prime}, t\right)=\int d x\left\{\frac{\omega^{+}(x)}{a^{+}}+\frac{\omega^{-}(x)}{a^{-}}\right\} E_{x}\left[d\left(x^{\varepsilon}(t), x^{\varepsilon^{\prime}}(t)\right)\right], \\
& Y_{2}\left(\varepsilon, \varepsilon^{\prime}, t\right)=\int d x\left\{\frac{\omega^{+}(x)}{a^{+}}+\frac{\omega^{-}(x)}{a^{-}}\right\} E_{x}\left[\varphi\left(x^{\varepsilon}(t), x^{\varepsilon^{\prime}}(t)\right)\right],
\end{aligned}
$$

we obtain

where

$$
Y_{1}\left(\varepsilon, \varepsilon^{\prime}, t\right) \leqq 2 c_{3}\|\omega\|_{\infty} \varepsilon t+\bar{c}(\omega) \int_{0}^{t} d s Y_{2}\left(\varepsilon, \varepsilon^{\prime}, s\right)
$$

$$
\bar{c}(\omega)=c(\omega)+a\left\{c\left(\frac{\omega^{+}}{a^{+}}\right)+c\left(\frac{\omega^{-}}{a^{-}}\right)\right\} .
$$

We now want to estimate $Y_{2}$ in terms of $Y_{1}$. Since

$$
\varphi(x, y)=\tilde{\varphi}(d(x, y))
$$

by the concavity of $\tilde{\varphi}$ and Jensen inequality,

$$
Y_{2}\left(\varepsilon, \varepsilon^{\prime}, t\right) \leqq 2 \tilde{\varphi}\left(Y_{1}\left(\varepsilon, \varepsilon^{\prime}, t\right)\right) .
$$

Let $h\left(x_{0}, t\right)$ be the solution of the following initial value problem $(B=2 \bar{c}(\omega))$ :

$$
\left.\begin{array}{rl}
\dot{z}(t) & =B \tilde{\varphi}(z(t)) \\
z(0) & =x_{0}>0 .
\end{array}\right\}
$$

Then if $x_{0}<1$,

$$
\begin{aligned}
& h\left(x_{0}, t\right)=x_{0}^{\exp (-B t)} \exp \left(1-e^{-B t}\right) \quad \text { if } \quad h<1 \\
& =1+B\left(t-t_{0}\right) \quad \text { if } h \geqq 1 \text {, }
\end{aligned}
$$

where

$$
t_{0}=\inf \left\{t \mid h\left(x_{0}, t\right)>1\right\} \text {, }
$$

and if $x_{0} \geqq 1$,

$$
h\left(x_{0}, t\right)=x_{0}+B t
$$

From (3.25) we have $\lim _{x_{0} \rightarrow 0} h\left(x_{0}, t\right)=0$ and since

$$
Y_{1}\left(\varepsilon, \varepsilon^{\prime}, t\right) \leqq h\left(2 c_{3}\|\omega\|_{\infty} t \varepsilon, t\right)
$$

by $(3.20)$ and (3.23) we obtain

$$
\lim _{\substack{\varepsilon \rightarrow 0 \\ \varepsilon^{\prime}<\varepsilon}} \sup _{\substack{\varepsilon^{\prime}<[0, T] \\ R}} \hat{R}\left(\omega_{t}^{\varepsilon}, \omega_{t}^{\varepsilon^{\prime}}\right)=0
$$

because $\hat{R}\left(\omega_{t}^{\varepsilon}, \omega_{t}^{\varepsilon^{\prime}}\right) \leqq Y_{1}\left(\varepsilon, \varepsilon^{\prime}, t\right)$.

Thus we obtain the existence of a family of weakly continuous limiting measures $\left\{\omega_{t}(d x)\right\}_{t \in \mathbb{R}} \in \mathscr{M}^{+}\left(a^{+}, a^{-}\right)$. By the estimate $\left\|\omega_{t}^{\varepsilon}\right\|_{\infty} \leqq\|\omega\|_{\infty}$ and $\left\|\omega_{t}^{\varepsilon}\right\|_{1}$ 
$\leqq\|\omega\|_{1}$, we conclude that $\omega_{t}(d x)$ is absolutely continuous with respect to the Lebesgue measure and denoting by $\omega_{t}$ its density,

$$
\left\|\omega_{t}\right\|_{1}=\|\omega\|_{1}, \quad\left\|\omega_{t}\right\|_{\infty} \leqq\|\omega\|_{\infty} .
$$

We claim that the map $\omega \rightarrow \omega_{t}$ is a weak solution of the NS equation. We first observe that $u(x, t)=\int K(x-y) \omega(y) d y$ exists because (3.28) and Lemma 3.1. Furthermore

$$
u(x, t)=\lim _{\varepsilon \rightarrow 0} u_{\varepsilon}(x, t)
$$

In fact

$$
\begin{aligned}
\left|u(x, t)-u_{\varepsilon}(x, t)\right| & =\left|\int\left\{K(x-y) \omega_{t}(y)-K_{\varepsilon}(x-y) \omega_{t}^{\varepsilon}(y)\right\} d y\right| \\
& \leqq\left|\int K(x-y)\left\{\omega_{t}(y)-\omega_{t}^{\varepsilon}(y)\right\} d y\right|+2 c_{3} \varepsilon\|\omega\|_{\infty} \\
& \leqq\left\{2 c_{3}(\varepsilon+\eta)\right\}\|\omega\|_{\infty}+\left|\int_{|x-y|>\eta} K(x-y)\left\{\omega_{t}(y)-\omega_{t}^{\varepsilon}(y)\right\} d y\right|
\end{aligned}
$$

The last term in (3.30) goes to zero as $\varepsilon \rightarrow 0$ by (3.27) and standard arguments. Thus (3.29) follows since $\eta$ is arbitrary.

Moreover

$$
\omega_{t}[(u \cdot \nabla) f]=\lim _{\varepsilon \rightarrow 0} \omega_{t}^{\varepsilon}\left[\left(u_{\varepsilon} \cdot \nabla\right) f\right]
$$

for all $f \in C_{1}\left(\mathbb{R}^{2}\right)$ such that $|\nabla f| \in L_{\infty}\left(\mathbb{R}^{2}\right)$.

Proof of (3.31).

$$
\begin{aligned}
\left|\omega_{t}[(u \cdot \nabla) f]-\omega_{t}^{\varepsilon}\left[\left(u_{\varepsilon} \cdot \nabla\right) f\right]\right| \leqq & \left|\omega_{t}[(u \cdot \nabla) f]-\omega_{t}^{\varepsilon}[(u \cdot \nabla) f]\right| \\
& +\left|\omega_{t}^{\varepsilon}[(u \cdot \nabla) f]-\omega_{t}^{\varepsilon}\left[\left(u_{\varepsilon} \cdot \nabla\right) f\right]\right| .
\end{aligned}
$$

The first term on the right hand side of (3.32) $\rightarrow 0$ because of (3.3) since $(u \cdot \nabla) f$ is a continuous and bounded function by virtue of (3.28) and Lemma 3.1.

The second term on the right hand side of

$$
\begin{aligned}
(3.32) \leqq & \|\nabla f\|_{\infty} \int d x\left|\omega_{t}^{\varepsilon}(x)\right|\left|u(x, t)-u_{\varepsilon}(x, t)\right| \\
\leqq & \|\nabla f\|_{\infty}\|\omega\|_{\infty} \int_{|x|<r}\left|u(x, t)-u_{\varepsilon}(x, t)\right| \\
& +\|\nabla f\|_{\infty} C(\omega) \int d x\left|\omega_{t}^{\varepsilon}(\infty)\right| X(\{|x| \geqq r\}),
\end{aligned}
$$

where $X(\{\})$ is the indicator of the set \{\} . Then, $\int_{|x|<r} \ldots d x \rightarrow 0$ because of (3.29) and the dominated convergence theorem. Hence by (3.3),

$$
\begin{aligned}
\limsup _{\varepsilon \rightarrow 0} 2^{\text {th }} \text { term of }(3.22) \leqq & \|\nabla f\|_{\infty} c(\omega) \int d x\left|\omega_{t}(x)\right| \\
& X(\{|x|>r\}) \leqq O(r),
\end{aligned}
$$

where

$$
\lim _{r \rightarrow \infty} O(r)=0
$$

because $\omega_{t} \in L_{1}\left(\mathbb{R}^{2}\right)$. This proves (3.31). 
By the uniform boundness (in $\varepsilon$ and $t$ on compact sets) of $\omega_{t}^{\varepsilon}[(u \cdot \nabla) f]$ and $\omega_{t}^{\varepsilon}(\Delta f)$ we obtain that $\omega_{t}$ satisfies the NS equation in the integral form and hence by weak continuity in the differential form (3.1).

Let $t \rightarrow \omega_{t}$ a weakly continuous family of $L_{1} \cap L_{\infty}\left(\mathbb{R}^{2}\right)$ functions such that $\left\|\omega_{t}\right\|_{1}$ $\leqq\left\|\omega_{0}\right\|_{1},\left\|\omega_{t}\right\|_{\infty} \leqq\left\|\omega_{0}\right\|_{\infty}$.

Defining

$$
u(x, t)=\int K(x-y) \omega_{t}(y) d y,
$$

we prove that the process solution of

$$
d x(t)=u(x(t), t) d t+\sigma d w(t)
$$

may be defined. To this purpouse let us introduce the Banach space $\mathscr{B}$ of all processes $\xi_{t}$ defined for $t \in[0, T]$ such that $\|\xi\|<\infty$ where

$$
\|\xi\|=\sup _{t \in[0, T]} E(d(\xi(t))), \quad d(x)=d(x, 0) .
$$

The processes starting almost surely form $x$ at time zero and satisfying

$$
d x^{\varepsilon}(t)=\bar{u}_{\varepsilon}\left(x^{\varepsilon}(t), t\right) d t+\sigma d w(t),
$$

where

$$
\bar{u}_{\varepsilon}(x, t)=\int K_{\varepsilon}(x-y) \omega_{t}(y) d y
$$

are a Cauchy sequence in $\mathscr{B}$. By Lemma 3.1 we have $\langle P=1\rangle$,

$$
\begin{aligned}
& d\left(x^{\varepsilon}(t)-x^{\varepsilon^{\prime}}(t)\right) \leqq \int_{0}^{t} d s \int \omega_{s}(d y)\left|K_{\varepsilon}\left(x^{\varepsilon}(s)-y\right)-K_{\varepsilon^{\prime}}\left(x^{\varepsilon}(s)-y\right)\right| \\
& \leqq \\
& \quad \int_{0}^{t} d s\left\{\int \omega_{s}(y)\left|K_{\varepsilon}\left(x^{\varepsilon}(s)-y\right)-K_{\varepsilon}\left(x^{\varepsilon^{\prime}}(s)-y\right)\right| d y\right. \\
& \left.\quad+\int \omega_{s}(y)\left|K_{\varepsilon}\left(x^{\varepsilon^{\prime}}(s)-y\right)-K_{\varepsilon^{\prime}}\left(x^{\varepsilon^{\prime}}(s)-y\right)\right| d y\right\} \\
& \leqq \\
& \quad \int_{0}^{t} d s c\left(\omega_{0}\right) \tilde{\varphi}\left(\left|x^{\varepsilon}(s)-x^{\varepsilon^{\prime}}(s)\right|\right)+c_{2}\left\|\omega_{0}\right\|_{\infty} \int_{|y| \leqq \varepsilon} d y|y|^{-1} .
\end{aligned}
$$

Proceeding as above we get $E\left(d\left(x^{\varepsilon}(t)-x^{\varepsilon^{\prime}}(t)\right)\right) \leqq h(0(\varepsilon), t)$ and hence the existence of a process such that $x(t)=\lim _{\varepsilon \rightarrow 0} x^{\varepsilon}(t)$ in $\mathscr{B}$. Moreover the differential of $x(t)$ is $u(x(t), t) d t$ $+\sigma d w$ as follows by the use of the same arguments leading to (3.41). To complete the proof it remains to show the uniqueness of the solution and of the process we have constructed. Let $\omega \rightarrow \bar{\omega}_{t}$ be another solution of the NS equation such that $\bar{\omega}_{t} \in L_{1} \cap L_{\infty}\left(\mathbb{R}^{2}\right)$. Proceeding as in the existence part one can prove that $\hat{R}\left(\bar{\omega}_{t}, \omega_{t}^{\varepsilon}\right) \rightarrow 0$ and hence the uniqueness follows. In the same way one proves the uniqueness of the process. If $\bar{x}(t)$ is another process satisfying (3.37), by (3.41) it follows that $\left\|\bar{x}-x^{\varepsilon}\right\| \rightarrow 0$ and this concludes the proof.

Proof of Lemma 3.1. By definition of $g_{\varepsilon}$ there exists a constant $c>0$ such that

$$
\left|\frac{\partial g_{\varepsilon}}{\partial x_{i}}(x)\right| \leqq \frac{c}{|x|}, \quad\left|\frac{\partial^{2} g_{\varepsilon}(x)}{\partial x_{i} \partial x_{j}}\right| \leqq \frac{c}{|x|^{2}} \text {. }
$$


Hence

$$
\begin{aligned}
& \int\left|\omega_{t}(y)\right|\left|\left(\nabla^{\perp} g_{\varepsilon}\right)(|x-y|)\right| d y \\
& \quad \leqq 2 c \int \frac{\left|\omega_{t}(y)\right|}{|x-y|} d y=2 c\left(\int_{|x+y| \leqq 1}+\int_{|x-y|>1}\right) \\
& \quad \leqq \operatorname{const}\left(A_{1}+A_{2}\right) .
\end{aligned}
$$

To prove (3.8) we put $r=\left|x-x^{\prime}\right|$ and $A=\{y|| x-y \mid \leqq 2 r\}$. If $r>1$ then (3.8) follows by (3.7). Otherwise

$$
\text { left hand side of }(3.8)=\int_{A}+\int_{\mathbb{R}^{2} \backslash A} d y\left(\left|\omega_{t}(y)\right|\left|K_{\varepsilon}(x-y)-K_{\varepsilon}\left(x^{\prime}-y\right)\right|\right) \text {. }
$$

We have

$$
\begin{aligned}
\int_{A} \ldots d y & \leqq c\left\|\omega_{t}\right\|_{\infty}\left\{\int_{A} \frac{d y}{|x-y|}+\int_{A} \frac{d y}{\left|x^{\prime}-y\right|}\right\} \\
& \leqq c\left\|\omega_{t}\right\|_{\infty}\left\{\int_{|x-y| \leqq 2 r} \frac{d y}{|x-y|}+\int_{\left|x^{\prime}-y\right| \leqq 3 r} \frac{d y}{\left|x^{\prime}-y\right|}\right\} \\
& \leqq \text { const }\left\|\omega_{t}\right\|_{\infty} r .
\end{aligned}
$$

On the other hand, for all $x^{\prime \prime}$ in the segment $\left(x, x^{\prime}\right)$ one has $\left|x^{\prime \prime}-y\right|>\frac{1}{2}|y-x|$, and hence

$$
\begin{aligned}
\int_{\mathbb{R}^{2} \backslash A} \ldots d y & \leqq 2 c r \int_{\mathbb{R}^{2} \backslash A} \frac{\left|\omega_{t}(y)\right|}{|y-x|^{2}} \\
& =2 c r \int_{2 r<|y-x|<2} \ldots d y+2 c r \int_{|y-x|>2} \ldots d y .
\end{aligned}
$$

The last integral is bounded by const $r\|\omega\|_{1}$ and finally

$$
\int_{2 r<|y-x|<2} \frac{\left|\omega_{t}(y)\right|}{|y-x|^{2}} d y \leqq \text { const }\left\|\omega_{t}\right\|_{\infty} \int_{2 r}^{2} \frac{d \varrho}{\varrho} .
$$

\section{The Mean Field Limit}

As we have seen in Sect. 1, the solutions of the vortex dynamics may be thought of as weak solutions of the Euler equation. This allows us to investigate the approximation problem stated in the Introduction as a continuity property with respect to the initial conditions. In presence of viscosity the situation is more complicated. This case will be discussed later.

We consider a system of $N$ vortices of intensity $a_{1}, a_{2} \ldots a_{N}$, interacting via a regularized potential :

$$
\left.\begin{array}{l}
\dot{x}_{i}^{N}(t)=\sum_{j=1}^{N} a_{j} K_{\varepsilon}\left(x_{i}^{N}(t)-x_{j}^{N}(t)\right) \\
x_{i}(0)=x_{i} \quad i=1 \ldots N .
\end{array}\right\}
$$


Defining

$$
\mu_{t}^{N}(d x)=\sum_{i=1}^{N} a_{i} \delta_{x_{i}^{N}(t)}
$$

with $\left\|\mu^{N \pm}\right\|=a^{ \pm}$, we have:

Theorem 4.1 (Mean field limit). Let $v=0$ and $\omega \in L_{1} \cap L_{\infty}\left(\mathbb{R}^{2}\right)$ such that $\omega d x \in \mathscr{M}\left(a^{+}, a^{-}\right)$. For all $\mu_{0}^{N}$ such that

$$
\lim _{N \rightarrow \infty} \mu_{0}^{N}(f)=\omega(f), \quad f \in \mathscr{C} \cap L_{\infty}\left(\mathbb{R}^{2}\right),
$$

and all sequence $\varepsilon=\varepsilon(N)$ such that

$$
\lim _{N \rightarrow \infty} \varepsilon(N)=0
$$

and

$$
\lim _{N \rightarrow \infty} \hat{R}\left(\mu_{0}^{N}, \omega\right) \exp \left[2 a L_{\varepsilon}\left(T+e^{2 a L_{\varepsilon} T}\right)\right]=0,
$$

then

$$
\lim _{N \rightarrow \infty} \mu_{t}^{N}(f)=\omega_{t}(f)
$$

where $\omega_{t}$ is the solution of the Euler equation with initial datum $\omega$.

Proof. Let $\varepsilon=\varepsilon(N)$ be a sequence satisfying (4.4). Defining $\omega_{t}^{N} \equiv \omega_{t}^{\varepsilon(N)}$ the solution of the regularized Euler problem with initial datum $\omega$ [see (2.6) for $v=0]$, we have:

$$
\begin{aligned}
& \hat{R}\left(\omega_{t}, \mu_{t}^{N}\right) \leqq \hat{R}\left(\omega_{t}, \omega_{t}^{N}\right)+\hat{R}\left(\omega_{t}^{N}, \mu_{t}^{N}\right), \\
& \hat{R}\left(\omega_{t}^{N}, \mu_{t}^{N}\right) \leqq \hat{R}\left(\omega_{t}^{N}, S_{t}^{\tilde{\mu}^{N}} \omega\right)+\hat{R}\left(S_{t}^{\tilde{\mu}^{N}} \omega, \mu_{t}^{N}\right) .
\end{aligned}
$$

Furthermore by (2.21) and Proposition 2.1

$$
\begin{gathered}
\hat{R}\left(\omega_{t}^{N}, S_{t}^{\tilde{\mu}^{N}} \omega\right) \leqq 2 a L_{\varepsilon} \exp 2 a L_{\varepsilon} T \int_{0}^{t} d s \hat{R}\left(\omega_{s}^{N}, \mu_{s}^{N}\right), \\
\hat{R}\left(S_{t}^{\tilde{\mu}^{N}} \omega, \mu_{t}^{N}\right) \leqq \hat{R}\left(\omega, \mu_{0}^{N}\right) \exp 2 a L_{\varepsilon} T .
\end{gathered}
$$

By (4.7) $-(4.9)$

$$
\hat{R}\left(\omega_{t}^{N}, \mu_{t}^{N}\right) \leqq \hat{R}\left(\omega, \mu_{0}^{N}\right) \exp \left[2 a L_{\varepsilon}\left(T+e^{2 a L_{\varepsilon} T}\right)\right]
$$

and hence Theorem 4.1 is proved by virtue of (3.3), that implies $\hat{R}\left(\omega_{t}, \omega_{t}^{N}\right) \underset{N \rightarrow \infty}{\longrightarrow} 0$, and assumption (4.4).

We consider now the viscous case. To simulate the solution of the NavierStokes equation in terms of a finite dimensional motion, a natural idea would be to study the vortex dynamics perturbed by a white noise.

More precisely, for all positive $\varepsilon>0$, we consider the following stochastic differential equation

$$
\begin{aligned}
& d X_{N}^{+}(t)=U_{\varepsilon}^{+}\left(X_{N}(t)\right) d t+\sigma d W(t), \\
& d X_{N}^{-}(t)=U_{\varepsilon}^{-}\left(X_{N}(t)\right) d t+\sigma d W(t),
\end{aligned}
$$


where

$$
\begin{gathered}
X_{N}^{ \pm}(t)=\left\{x_{1}^{ \pm}(t) \ldots x_{N}^{ \pm}(t)\right\}, x_{i}^{+}, x_{i}^{-}(t) \in \mathbb{R}^{2}, \quad i=1 \ldots N, \\
X_{N}(t)=\left(X_{N}^{+}(t), X_{N}^{-}(t)\right) \in\left(\mathbb{R}^{2}\right)^{2 N}, \\
U_{\varepsilon}^{ \pm}\left(Y_{N}\right)_{i}=N^{-1} \sum_{j=1}^{N}\left\{a^{+} K_{\varepsilon}\left(y_{i}^{ \pm}-y_{j}^{+}\right)-a^{-} K_{\varepsilon}\left(y_{i}^{ \pm}-y_{j}^{-}\right)\right\}, \\
i=1 \ldots N, Y_{N}=\left(Y_{N}^{+}, Y_{N}^{-}\right), Y_{N}^{ \pm}=\left\{y_{1}^{ \pm} \ldots y_{N}^{ \pm}\right\}, \\
\frac{\sigma^{2}}{2}=v, \sigma>0 ;
\end{gathered}
$$

and $W$ is the standard Wiener process in $\mathbb{R}^{2 N}$.

Equation (4.11) describes the motion of $N$ identical vortices with positive circulation given by $a^{+} N^{-1}$ and $N$ identical vortices with negative circulation given by $-a^{-} N^{-1}$. (We assume all vortices of the same intensity for the sake of simplicity.)

We fix a sequence of positive numbers $\varepsilon=\varepsilon(N)$ such that $\lim _{N \rightarrow \infty} \varepsilon(N)=0$. The process $X_{N}\left(t, X_{N}\right)=\left\{x_{i}^{N \pm}\left(t, X_{N}\right)\right\}_{i=1}^{N}$ solution of (4.11), starting almost surely from $X_{N}=\left(X_{N}^{+}, X_{N}^{-}\right)$is uniquely determined by $a^{ \pm}, N$, and $X_{N}$.

We define the following family of measures in $\mathscr{M}\left(a^{+}, a^{-}\right)$for $t \in[0, T]$ :

$$
\mu_{t}\left(f ; X_{N}\right)=\frac{1}{N} E\left[\sum_{i=1}^{N} a^{+} f\left(x_{i}^{N+}\left(t, X_{N}\right)\right)-a^{-} f\left(x_{i}^{N-}\left(t, X_{N}\right)\right)\right]
$$

$f$ continuous and bounded.

In analogy with the Euler case the measure $\mu_{t}\left(\cdot ; X_{N}\right)$ is expected to be an approximation of every solution of the NS equation with a suitable choice of $X_{N}$. The main difference with the $v=0$ case is that $\mu_{t}\left(\cdot ; X_{N}\right)$ is not a weak solution of the NS equation. This because a tagged vortex moves in a velocity field that is a random variable given by the random positions of all other vortices, while in the NS equation any path is determined by a velocity field that is a sure variable obtained by the solution of the same equation. Nevertheless one can hope that a phenomenon (known in kinetic theory as "propagation of chaos") could occur in this case also. This means that when $N$ is large and the intensity of any vortex very small, the velocity field generated by typical vortex paths is very similar to the velocity field computed on the average positions.

McKean proved in [8] propagation of chaos and mean field limit for a system of particles interacting via a smooth and bounded potential. He assumed that at time zero, the random variables $x_{1} \ldots x_{N}$ are identically distributed. Thus his result cannot be directly applied to our purpose.

We introduce the space $\Gamma=\left(\mathbb{R}^{2} \times \mathbb{R}^{2}\right)^{N}$ together with the $\sigma$-algebra $\mathscr{B}_{\infty}$ of all Borel sets with respect to the product topology. Given $\omega \in L_{1} \cap L_{\infty}\left(\mathbb{R}^{2}\right)$ such that $\omega d x \equiv \omega(d x) \in \mathscr{M}\left(a^{+}, a^{-}\right)$, we define the measure $\omega_{\infty}=\left(\frac{\omega^{+}}{a^{+}} \times \frac{\omega^{-}}{a^{-}}\right)^{\mathbb{N}}$ on $\left(\Gamma, \mathscr{B}_{\infty}\right)$. Finally, given $X \in \Gamma$ we denote by $X_{N}^{ \pm}=\left(x_{1}^{ \pm} \ldots x_{N}^{ \pm}\right)$its first $N$ components. 
Theorem 4.2. Let $v>0, \omega \in L_{1} \cap L_{\infty}\left(\mathbb{R}^{2}\right)$ such that $\omega(d x) \in \mathscr{M}\left(a^{+}, a^{-}\right)$. Then, for $\omega_{\infty}$-a.a. $X \in \Gamma$, there exists a sequence such that

$$
\lim _{N \rightarrow \infty} \mu_{0}\left(f ; X_{N}\right) \equiv \lim _{N \rightarrow \infty} N^{-1} \sum_{i=1}^{N}\left\{a^{+} f\left(x_{i}^{+}\right)-a^{-} f\left(x_{i}^{-}\right)\right\}=\omega(f)
$$

and

$$
\lim _{N \rightarrow \infty} \mu_{t}\left(f ; X_{N}\right)=\omega_{t}(f), \quad t>0
$$

for all $f \in\left(\mathscr{C} \cap L_{\infty}\right)\left(\mathbb{R}^{2}\right)$, where $\omega_{t}$ denotes the unique solution of NS equation with initial datum $\omega$.

Proof. Without loss of generality we put $\sigma=1$.

Let $\Omega=C([0, T])$ be the set of all $\mathbb{R}^{2}$-valued continuous functions defined in $[0, T] . \Omega$ is a metric space with metric function given by $d(\xi, \eta)=\sup _{t \in[0, T]} d(\xi(t), \eta(t))$. We denote by $\Sigma$ the $\sigma$-algebra of the Borel sets in $\Omega$ and by $\Sigma^{\infty}$ the $\sigma$-algebra of the Borel sets in $\Omega^{\infty} \equiv(\Omega \times \Omega)^{\mathbb{N}}$.

Consider now $X^{N}(t, X ; \tilde{\varrho})=\left\{x_{i}^{N \pm}(t, X ; \varrho)\right\}_{i=1}^{N}, \varrho \varrho \in \Omega^{\infty}$, the solutions of the integral equation:

$$
x_{i}^{N \pm}(t, X ; \varrho)=x_{i}^{ \pm}+\varrho_{i}^{ \pm}(t)+\int_{0}^{t} d s U_{\varepsilon}^{ \pm}\left(X_{N}(t, X ; \tilde{\varrho})\right)_{i},
$$

where $X=\left\{x_{i}^{ \pm}\right\}_{i=1}^{\infty} \in \Gamma$. Then $X^{N}(t, X ; \varrho)$ is, for all $t$, a family of random variables in $\left(\Omega^{\infty}, \Sigma^{\infty}, P_{0}^{\infty}\right)$, where $P_{0}^{\infty}=\left(P_{0} \times P_{0}\right)^{\mathbb{N}}$ and $P_{0}$ is the Wiener measure (supported on the trajectories of $\Omega$ starting from 0$)$. Obviously $x_{i}^{N \pm}(t, X ; \tilde{\varrho})$ depends only on $X_{N}$ and $\varrho_{1} \ldots \varrho_{N}$. The process (4.16) is a realization of the solution of (4.11). Such a solution may also be thought of as realized on $\left(\Omega^{\infty}, \Sigma^{\infty}, P_{X}^{\infty}\right)$, where $P_{X}^{\infty}=\prod_{i=1}^{\infty}\left(P_{x_{t}^{+}} \times P_{x_{i}^{-}}\right)$and where $\int P_{x}(d \eta) f(\eta)=\int P_{0}(d \eta) f(\eta-x)$. Denoting it by $\left\{x_{i}^{N \pm}(t, \tilde{\xi})\right\}$, then

$$
x_{i}^{N \pm}(t, \tilde{\xi})=\xi_{i}^{ \pm}(t)+\int_{0}^{t} d s U_{\varepsilon}^{ \pm}\left(X^{N}(t, \tilde{\xi})\right)_{i}
$$

Let us define a family of Borel measures on $\mathbb{R}^{2}$ indexed by $\tilde{\xi} \in \Omega^{\infty}$ :

$$
\mu_{t}^{N}(f ; \tilde{\xi})=N^{-1} \sum_{i=1}^{N}\left[a^{+} f\left(x_{i}^{N+}(t, \tilde{\xi})\right)-a^{-} f\left(x_{i}^{N-}(t, \tilde{\xi})\right)\right] .
$$

Then

$$
\mu_{t}\left(f, X_{N}\right)=\int P_{X}^{\infty}(d \tilde{\xi}) \mu_{t}^{N}(f ; \tilde{\xi})
$$

Finally we introduce the two dimensional process $x^{N}(t, \tilde{\xi} ; \eta)$ defined on $\left(\Omega, \Sigma, P_{x}\right)$, satisfying

$$
x^{N}(t, \tilde{\xi} ; \eta)=\eta(t)+\int_{0}^{t} \tilde{u}_{\varepsilon}\left(x^{N}(s, \tilde{\xi} ; \eta), s\right) d s
$$


where

$$
\tilde{u}_{\varepsilon}(y, s)=\int K_{\varepsilon}(y-x) \mu_{s}^{N}(d x ; \tilde{\xi}) .
$$

We have to estimate $\hat{R}\left(\omega_{t}, \mu_{t}\left(\cdot ; X_{N}\right)\right)$. Then

$$
\hat{R}\left(\omega_{t}, \mu_{t}\left(\cdot ; X_{N}\right)\right) \leqq \hat{R}\left(\omega_{t}, \omega_{t}^{N}\right)+\hat{R}\left(\omega_{t}^{N}, \mu_{t}\left(\cdot ; X_{N}\right)\right),
$$

where $\omega_{t}^{N} \equiv \omega_{t}^{\varepsilon(N)}$. If $\varepsilon=\varepsilon(N) \rightarrow 0$ when $N \rightarrow \infty$ the first term in the right hand side of (4.22) goes to zero by (3.3). To estimate the second term in the right hand side of (4.22), we try an estimate for each trajectory.

$$
\hat{R}\left(\omega_{t}^{N}, \mu_{t}^{N}(\cdot, \tilde{\xi})\right) \leqq \hat{R}\left(\omega_{t}^{N}, \tilde{\omega}_{t}^{N}(\cdot, \tilde{\xi})\right)+\hat{R}\left(\tilde{\omega}_{t}^{N}(\cdot ; \xi), \mu_{t}^{N}(\cdot ; \tilde{\xi})\right),
$$

where

$$
\tilde{\omega}_{t}^{N}(f ; \tilde{\xi})=\int d x \omega(x) \int P_{x}(d \eta) f\left(x^{N}(t, \tilde{\xi} ; \eta)\right)
$$

By virtue of (2.21) we have

$$
\hat{R}\left(\omega_{t}^{N}, \tilde{\omega}_{t}^{N}(\cdot ; \tilde{\xi})\right) \leqq 2 a L_{\varepsilon} e^{2 a L_{\varepsilon} T} \int_{0}^{t} d s \hat{R}\left(\omega_{s}^{N}, \mu_{s}^{N}(\cdot ; \tilde{\xi})\right) .
$$

Furthermore, realizing that $x^{N \pm}\left(t, \tilde{\xi} ; \xi_{i}^{ \pm}\right)=x_{i}^{N \pm}(t, \tilde{\xi})$, we have

$$
\mu_{t}^{N}(f ; \tilde{\xi})=\int v_{N}(d \eta ; \tilde{\xi}) f\left(x^{N}(t, \tilde{\xi} ; \eta)\right),
$$

where

$$
\int v_{N}(d \eta ; \tilde{\xi}) F(\eta)=N^{-1} \sum_{i=1}^{N}\left\{a^{+} F\left(\xi_{i}^{+}\right)-a^{-} F\left(\xi_{i}^{-}\right)\right\}
$$

for all continuous and bounded $F$.

Thus, to estimate the second term in the right hand side of (4.23), we have to compare different trajectories of the process (4.20). We have

$$
\begin{aligned}
& d\left(x^{N}\left(t, \tilde{\xi} ; \eta_{1}\right), x^{N}\left(t, \tilde{\xi} ; \eta_{2}\right)\right) \leqq\left|\eta_{1}(t)-\eta_{2}(t)\right| \\
& \quad+\mid \int_{0}^{t} d s \int \mu_{s}^{N}(d x ; \tilde{\xi})\left[K_{\varepsilon}\left(x\left(s, \tilde{\xi} ; \eta_{1}\right)-x\right)-K_{\varepsilon}\left(x\left(s, \tilde{\xi} ; \eta_{2}\right)-x\right] \mid .\right.
\end{aligned}
$$

This implies

$$
d\left(x^{N}\left(t, \tilde{\xi} ; \eta_{1}\right), x^{N}\left(t, \tilde{\xi} ; \eta_{2}\right)\right) \leqq\left|\eta_{1}(t)-\eta_{2}(t)\right| \exp 2 L_{\varepsilon} a T
$$

and hence

$$
d\left(x^{N}\left(t, \tilde{\xi} ; \eta_{1}\right), x^{N}\left(t, \tilde{\xi} ; \eta_{2}\right)\right) \leqq d\left(\eta_{1}, \eta_{2}\right) e^{2 L_{\varepsilon} a T}
$$

Denoting by $v_{N}^{ \pm}(\cdot ; \tilde{\xi})$ the positive and negative part of $v_{N}(\cdot ; \tilde{\xi})$, putting $\lambda^{ \pm}(d \eta)$ $=\int \omega^{ \pm}(x) d x \int P_{x}(d \eta)$ and $\lambda=\lambda^{+}-\lambda^{-}$, we define joint representations $\tilde{Q}^{ \pm} \in \mathscr{C}\left(\left(a^{ \pm}\right)^{-1} \mu_{t}^{N \pm}(\cdot ; \tilde{\xi}), \quad\left(a^{ \pm}\right)^{-1} \tilde{\omega}_{t}^{N \pm}(\cdot ; \tilde{\xi})\right)$ via joint representations $Q^{ \pm} \in \mathscr{C}\left(\left(a^{ \pm}\right)^{-1} v_{N}^{ \pm}(\cdot, \tilde{\xi}),\left(a^{ \pm}\right)^{-1} \lambda^{ \pm}\right) \equiv \mathscr{A}$ :

$$
\int \tilde{Q}^{ \pm}(d x, d y) f(x, y)=\int Q^{ \pm}(d \eta, d \varrho) f\left(x^{N}(t, \tilde{\xi} ; \eta), x^{N}(t, \tilde{\xi} ; \varrho)\right) .
$$


Hence, still denoting the KR distance in $\mathscr{M}(\Omega ; 1)$ by $R_{1}$ and in $\mathscr{M}\left(\Omega ; a^{+}, a^{-}\right)$by $\hat{R}$, we have:

$$
\begin{aligned}
& R_{1}\left(\frac{\tilde{\omega}^{N \pm}}{a^{ \pm}}(\cdot, \tilde{\xi}), \frac{\mu_{t}^{N \pm}}{a^{+}}(\cdot, \tilde{\xi})\right) \\
& \quad \leqq \inf _{Q^{ \pm} \in \mathscr{A}} \int \tilde{Q}^{ \pm}(d x, d y) d(x, y) \\
& \quad=\inf _{Q^{ \pm} \in \mathscr{A}} \int Q^{ \pm}(d \eta, d \varrho) d\left(x^{N}(t, \tilde{\xi} ; \eta), x^{N}(t, \tilde{\xi} ; \varrho)\right) \\
& \leqq \\
& \quad \inf _{Q^{ \pm} \in \mathscr{A}} \int Q^{ \pm}(d \eta, d \varrho) \exp 2 L_{\varepsilon} a T d(\eta, \varrho) \\
& \quad=\exp 2 L_{\varepsilon} a T R_{1}\left(\frac{v_{N}^{ \pm}(\cdot, \xi)}{a^{ \pm}}, \frac{\lambda^{ \pm}}{a^{ \pm}}\right)
\end{aligned}
$$

and hence

$$
\hat{R}\left(\tilde{\omega}_{t}^{N}(\cdot, \tilde{\xi}), \mu_{t}^{N}(\cdot, \tilde{\xi})\right) \leqq e^{2 L_{\varepsilon} a T} \hat{R}\left(v_{N}(\cdot, \tilde{\xi}), \lambda\right)
$$

Defining $\lambda^{\infty}=\left(\frac{\lambda^{+}}{a^{+}} \times \frac{\lambda^{-}}{a^{-}}\right)^{\mathbb{N}}$ as a probability measure on $\left(\Omega^{\infty}, \Sigma^{\infty}\right)$ we prove that for $\lambda_{\infty}$-almost all $\widetilde{\xi} \in \Omega^{\infty}$

$$
\underset{N \rightarrow \infty}{\operatorname{weak}} \lim v_{N}^{ \pm}(d \eta ; \tilde{\xi})=\lambda^{ \pm}(d \eta)
$$

To prove (4.34) we introduce the countable family of random variables on $\left(\Omega, \sum, \lambda^{ \pm}\left(a^{ \pm}\right)^{-1}\right)$ :

$$
\begin{gathered}
X_{t_{1} \ldots t_{k}}^{A_{1} \ldots A_{k}}=X\left(\left\{\eta \mid \eta\left(t_{1}\right) \in A_{i}, i=1 \ldots k\right\}\right), \\
\left|\eta\left(t_{i}\right)-\eta\left(t_{j}\right)\right|^{4},
\end{gathered}
$$

where $t_{i}$ are rational instants in $[0, T]$ and $A_{i}=\left[a_{i}, b_{i}\right), a_{i}, b_{i} \in \mathbb{R}^{2} ; a_{i}^{\alpha}, b_{i}^{\alpha}, \alpha=1,2$ are rational numbers.

By the strong law of large numbers, there exists a set $\hat{\Omega}^{\infty} \subset \Omega^{\infty}$ such that $\lambda^{\infty}\left(\hat{\Omega}^{\infty}\right)=1$ and for all $\tilde{\xi} \in \hat{\Omega}^{\infty}$

$$
\begin{aligned}
\frac{v_{N}^{ \pm}}{a^{ \pm}}\left(X_{t_{1} \ldots t_{k}}^{A_{1} \ldots A_{k}} ; \tilde{\xi}\right) & =N^{-1} \sum_{i=1}^{N} X_{t_{1} \ldots t_{k}}^{A_{1} \ldots A_{k}}\left(\xi_{i}^{ \pm}\right) \underset{N \rightarrow \infty}{\longrightarrow} \frac{\lambda^{ \pm}}{a^{ \pm}}\left(X_{t_{1} \ldots t_{k}}^{A_{1} \ldots A_{k}}\right) \\
\frac{v_{N}^{ \pm}}{a^{ \pm}}\left(\left|\xi\left(t_{k}\right)-\xi\left(t_{j}\right)\right|^{4}\right) & =N^{-1} \sum_{i=1}^{N}\left|\xi_{i}\left(t_{k}\right)-\xi_{i}\left(t_{j}\right)\right|^{4} \\
& \rightarrow \frac{\lambda^{ \pm}}{a^{ \pm}}\left(\left|\xi\left(t_{k}\right)-\xi\left(t_{j}\right)\right|^{4}\right)=\mathrm{const}\left|t_{k}-t_{j}\right|^{2} .
\end{aligned}
$$

Combining Theorem 2, p. 514 and Remark 1, p. 513 of [10], we conclude from (4.37) and (4.38), that the sequence $v_{N}^{ \pm}(\cdot ; \tilde{\xi})$ is relatively weakly compact for $\tilde{\xi} \in \hat{\Omega}^{\infty}$ and from (4.37) that it has a unique limit point, i.e. $\lambda^{ \pm}$. Thus (4.34) is proved.

Furthermore, defining for $X \in \Gamma$

$$
\hat{\Omega}^{\infty}(X)=\left\{\tilde{\eta} \in \hat{\Omega}^{\infty} \mid\left\{\eta_{i}^{+}(0), \eta_{i}^{-}(0)\right\}_{i=1}^{\infty}=X\right\},
$$


there exists a set $\hat{\Gamma}$ such that $\omega^{\infty}(\hat{\Gamma})=1$ and if $X \in \hat{\Gamma}$ :

$$
P_{X}^{\infty}(\hat{\Omega}(X))=1 \text {. }
$$

(4.40) follows by the identity (it can be verified by a direct computation on a function $F$ depending only on a finite number of trajectories):

$$
\int \lambda^{\infty}(d \tilde{\eta}) F(\tilde{\eta})=\int \omega^{\infty}(d X) \int P_{X}(d \tilde{\eta}) F(\tilde{\eta}) .
$$

In particular

$$
\int \lambda^{\infty}(d \tilde{\eta}) X\left(\left\{\tilde{\eta} \in \hat{\Omega}^{\infty}\right\}\right)=\int \omega^{\infty}(d X) P_{X}^{\infty}\left(\hat{\Omega}^{\infty}(X)\right),
$$

that implies (4.40).

Then by (4.23), (4.25), and (4.33)

$$
\begin{aligned}
\hat{R}\left(\omega_{t}^{N}, \mu_{t}^{N}(\cdot ; \tilde{\xi})\right) \leqq & \left(\exp 2 L_{\varepsilon} T\right) \\
& \cdot\left\{\hat{R}\left(v_{N}(\cdot, \tilde{\xi}), \lambda\right)+2 L_{\varepsilon} a \int_{0}^{t} d s \hat{R}\left(\omega_{s}^{N}, \mu_{s}^{N}(\cdot, \tilde{\xi})\right)\right\} .
\end{aligned}
$$

Hence

$$
\hat{R}\left(\omega_{t}^{N}, \mu_{t}^{N}(\cdot ; \tilde{\xi})\right) \leqq \hat{R}\left(v_{N}(\cdot, \tilde{\xi}), \lambda\right) \exp 2 L_{\varepsilon} a\left(T+e^{2 a L_{\varepsilon} T}\right) .
$$

For all $Q_{\tilde{\xi}} \in \mathscr{C}\left(\frac{\omega_{t}^{N \pm}}{a^{ \pm}}, \frac{\mu_{t}^{N \pm}}{a^{ \pm}}(\cdot, \tilde{\xi})\right)$, then

$$
\int P_{X}^{\infty}(d \tilde{\xi}) Q_{\tilde{\xi}}(d x, d y) \in \mathscr{C}\left(\frac{\omega_{t}^{N \pm}}{a^{ \pm}}, \frac{\mu_{t}^{N \pm}}{a^{ \pm}}(\cdot, X)\right),
$$

hence, taking the expectation with respect to $P_{X}^{\infty}$, for $X \in \hat{\Gamma}$, we have

$$
\begin{aligned}
\hat{R}\left(\omega_{t}^{N}, \mu_{t}^{N}(\cdot ; X)\right) & \leqq \int P_{X}^{\infty}(d \tilde{\xi}) \hat{R}\left(\omega_{t}^{N}, \mu_{t}^{N}(\cdot ; \tilde{\xi})\right) \\
& \leqq O_{N}(X)\left\{\exp 2 L_{\varepsilon} a\left(T+e^{2 a L_{\varepsilon} T}\right)\right\},
\end{aligned}
$$

where $O_{N}(X)=\int P_{X}(d \tilde{\xi}) \hat{R}\left(v_{N}(\cdot, \tilde{\xi}), \lambda\right)$. By the dominated convergence theorem and (4.34) $O_{N}(X) \rightarrow 0$ and this achieves the proof for a suitable choice of $\varepsilon=\varepsilon(N)$ by virtue of (4.22).

We conclude this section with some comments.

We have not fully exploited the stochastic nature of the NS equation, since we were interested to give an unified approach to the NS and E equations. One would expect that the regularizing properties of the diffusion processes would improve the estimate in a $v$ dependent way.

It would be interesting to obtain the mean field limit starting directly from the unregularized problem (without the selfenergy factor) and by means of a constructive procedure in the case $v>0$. As a first step one needs to study the $N$ vortex process. It has to be proved that collapses between two vortices of opposite sign (that do happen), are exceptional in the sense of the Lebesgue measure, so that one can hope that the finite dimensional processes make sense. A result in this direction has been obtained for bounded domains [11].

Other kinds of systems may be treated with techniques similar to that used in this paper. For example a three dimensional fluid with particular symmetries 
admits a vortex description. A fluid in which the velocities field has a cyclindrical symmetry and lies in a plane containing the symmetry axis preserves the same structure at any time. Thus this system may be described in terms of a two dimensional fluid in half plane.

Finally, an interesting problem is the study of the vortex theory and the mean field limit in bounded regions. In this case the Green's function has to be modified and the right boundary conditions for $\mathrm{E}$ and NS equations have to be taken into account. The Euler case follows from our analysis with minor modification while the Navier-Stokes case seems to be more complicated. This is because the right boundary conditions on the velocity (i.e. $U=0$ on the boundary) imply rather complicated boundary conditions on the vorticity.

\section{References}

1. Friedrichs, K.O.: Special topics in fluid dynamics. New York: Gordon and Breach 1966

2. Hald, O., Mauceri Del Prete, V.: Math. Comput. 32, 791 (1978)

3. Saffman, P.G., Baker, G.R.: Ann. Rev. Fluid Mech. 11, 95 (1979)

4. Kato, T.: Arch. Rat. Mech. Anal. 25, 95 (1967)

5. Inue, A., Funaki, T.: Commun. Math. Phys. 65, 83 (1979)

6. Martinelli, F., Micheli, L.: Private communication

7. Dobrushin, R.L.: Sov. J. Funct. Anal. 13, 115 (1979)

8. McKean, H.P.: Propagation of chaos for a class of nonlinear parabolic equations, Lecture in differential equations, Vol. 2, Aziz, A.K. (ed.). Princeton, NJ : Van Nostrand 1969

9. Dobrushin, R.L.: Teor. Veroyatn. Priloz. 15, 469 (1970)

10. Gihman, I.I., Skorohod, A.V.: Introduction a la theorie des processus aleatoires. Moskow: MIR 1980

11. Dürr, D., Pulvirenti, M.: On the vortex flow in bounded domains. Commun. Math. Phys. (in press) (1982)

Communicated by J. Glimm

Received November 8, 1981 
